\title{
Some discussion on the existence of common fixed points for a pair of maps
}

\author{
Deepesh K Patel ${ }^{1}$, Poom Kumam² ${ }^{2 *}$ and Dhananjay Gopal ${ }^{1}$
}

\author{
"Correspondence: \\ poom.kum@kmutt.ac.th \\ 2Department of Mathematics, \\ Faculty of Science, King Mongkut's \\ University of Technology Thonburi \\ (KMUTT), 126 Pracha Uthit Rd., Bang \\ Mod, Thung Khru, Bangkok, 10140, \\ Thailand \\ Full list of author information is \\ available at the end of the article
}

\begin{abstract}
In this paper, the concepts of conditionally sequential absorbing and pseudo-reciprocal continuous maps are introduced in connection to giving a brief discussion on the role of various types of commutativity (e.g., weakly compatible, occasionally weakly compatible, subcompatible, pseudo-compatible, etc.) and continuity-type conditions (e.g., reciprocal, weak reciprocal, $g$-reciprocal, conditionally reciprocal, subsequential and sequential continuity of type $\left(A_{g}\right)$ and $\left.\left(A_{f}\right)\right)$ in the context of existence of common fixed points of a pair of maps. Here, the utility of newly introduced maps (i.e., conditionally sequential absorbing and pseudo-reciprocal continuous) in view of common fixed points for a pair of maps satisfying contractive as well as nonexpansive Lipschitz-type conditions is shown. MSC: $47 \mathrm{H} 10 ; 54 \mathrm{H} 25$

Keywords: common fixed point; conditionally sequential absorbing; pseudo-reciprocal continuity
\end{abstract}

\section{Introduction and preliminaries}

The classical results of Banach [1] (see also [2]) and Edelstein [3] have been the inspiration for many researchers working in the area of metric fixed point theory. In 1976, Jungck [4] generalized the Banach contraction principle by introducing the idea of commuting maps and settled the historical open problem that a pair of commuting and continuous selfmappings on the unit interval $[0,1]$ need not have a common fixed point $[5,6]$. This result of Jungck [4] made foundation to study and investigate common fixed points and their applications in various other branches of mathematical sciences in the last five decades. Since then many fixed point theorists have attempted to find weaker forms of commutativity and continuity that may ensure the existence of a common fixed point for a pair of selfmappings on a metric space. Systematic observations and comparison of commutativitytype mappings are available in [7].

Proving a common fixed point for mappings satisfying Banach-type contractive conditions involves the following steps: step one is to show that there exists a Cauchy sequence which converges to a point in $X$ (where $X$ is complete); the second step is to show the existence of a coincidence point by assuming suitable weaker forms of commutativity and continuity conditions; and step three automatically gives rise to the fact that this coincidence point is a unique common fixed point due to the contractive condition. Observing carefully step two, one finds that showing the existence of a coincidence point for involved maps is nothing but assuming the existence of a coincidence point itself by a suitable choice of weaker forms of commutativity and continuity conditions (see, for instance, [8-16]).

() 2013 Patel et al.: licensee Springer. This is an Open Access article distributed under the terms of the Creative Commons Attribution License (http://creativecommons.org/licenses/by/2.0), which permits unrestricted use, distribution, and reproduction in any medium, provided the original work is properly cited. 
Keeping the above facts in mind, Jungck and Rhoades [17] utilized the notion of occasionally weakly compatible maps introduced in [18] (as a generalization of weakly compatible maps) for those pairs which do have at least one coincidence point where the maps commute (it is well known that a pair of maps without a coincidence point is always vacuously weakly compatible) and obtained fixed point theorems for such maps.

On the other hand, Singh and Mishra in [19] illustrated a technique to prove the existence of a coincidence point without assuming continuity and commutativity-type conditions. Whereas the result of Suzuki and Pathak [20] does not involve any continuity-type conditions to prove the existence of a coincidence point as well as a common fixed point for a pair of maps (but they used weaker forms of commutativity conditions). It is also worth mentioning that Suzuki and Pathak [20] did not provide any illustrative examples to discuss and highlight the above facts. It is also important to note that none of the results of Jungck [9], Singh and Mishra [19] and Suzuki and Pathak [20] can be obtained from each other due to their different characteristics. These facts are illustrated in this paper via Example 2.6 (p.10).

Motivated by the works of Jungck and Rhoades [17], Bouhadjera and Thobie [21] (respectively Hussain et al. [22] and Sintunavarat and Kumam [23]) introduced the notion of subcompatible maps (respectively the notions of occasionally weakly $\mathcal{J H}$ operator and occasionally weakly biased maps) as generalization of occasionally weakly compatible maps and obtained fixed point theorems for such maps. However, Dorić et al. in [24] (respectively Alghamdi et al. [25]) showed that in the event of a pair of single-valued maps, the notion of occasionally weakly compatible (respectively occasionally weakly $\mathcal{J H}$ operator and occasionally weakly biased maps) reduces to weak compatibility due to the unique coincidence point of the involved maps, which is always ensured by underlying contractive conditions. Hence weak compatibility remains the minimal commutativity condition for the existence of a common fixed point for a contractive pair of maps. In view of these, the various results for occasionally weakly compatible maps (occasionally weakly $\mathcal{J H}$ operator and occasionally weakly biased maps) obtained in [17, 21, 22, 26-30], which were used under contractive conditions, do not yield real generalizations (see also [31, 32]). Considering these facts, Pant and Pant [33] (see also [34]) redefined the concept of occasionally weakly compatibility by introducing the idea of conditionally commuting maps which constitute a proper setting in the context of studying non-unique common fixed points for a pair of maps.

Possibly the first common fixed point theorem (respectively fixed point theorem) without any continuity requirement was established by Pant $[12,35]$ when he introduced the idea of noncompatible and reciprocal continuous maps. (However, the origin of metric fixed point theory for a single mapping without continuity requirement can be traced back to Kannan [36].) Recently, Pant et al. [37] and Pant and Bisht [38] generalized the notion of reciprocal continuity by introducing weak reciprocal continuity and conditionally reciprocal continuity and utilized the same to obtain some common fixed point theorems. In this connection, the recent paper of Gopal et al. [39] is also readable.

Motivated by the results of Pant and Bisht $[38,40]$, we introduce the concept of conditionally sequential absorbing and pseudo-reciprocal continuous maps, which allows us to give a comparative study of various types of commutativity conditions (e.g., compatible, weakly compatible, occasionally weakly compatible, conditionally commuting, pseudocompatible) and continuity-type conditions (e.g., reciprocal, weak reciprocal, $g$-reciprocal, 
conditionally reciprocal, subsequential and sequential continuity of type $\left(A_{g}\right)$ and $\left.\left(A_{f}\right)\right)$ with these newly introduced notions in the context of existence of common fixed points of a pair of maps.

The following relevant known definitions (and results) will be needed in our subsequent discussion. A pair $(f, g)$ of self-mappings defined on a metric space $(X, d)$ is said to be

(i) compatible [9] iff $\lim _{n} d\left(f g x_{n}, g f x_{n}\right)=0$ whenever $\left\{x_{n}\right\}$ is a sequence in $X$ such that $\lim _{n} f x_{n}=\lim _{n} g x_{n}=t$ for some $t$ in $X$.

It is clear from the above definition that $f$ and $g$ will be noncompatible [35] if there exists a sequence $\left\{x_{n}\right\}$ in $X$ such that $\lim _{n} f x_{n}=\lim _{n} g x_{n}=t$ for some $t$ in $X$, but $\lim _{n} d\left(f g x_{n}, g f x_{n}\right)$ is either nonzero or non-existent;

(ii) $f$-compatible [41] if $\lim _{n} d\left(f g x_{n}, g g x_{n}\right)=0$ whenever $\left\{x_{n}\right\}$ is a sequence in $X$ such that $\lim _{n} f x_{n}=\lim _{n} g x_{n}=t$ for some $t$ in $X$;

(iii) $g$-compatible [41] if $\lim _{n}\left(f x_{n}, g f x_{n}\right)=0$ whenever $\left\{x_{n}\right\}$ is a sequence in $X$ such that $\lim _{n} f x_{n}=\lim _{n} g x_{n}=t$ for some $t$ in $X$;

(iv) weakly compatible [42] if the mappings commute at their coincidence points, i.e., $f x=g x$ for some $x \in X$ implies $f g x=g f x$;

(v) occasionally weakly compatible [17] if there exists a point $x$ in $X$ that is a coincidence point of $f$ and $g$ at which $f$ and $g$ commute;

(vi) subcompatible [21] iff there exists a sequence $\left\{x_{n}\right\}$ in $X$ such that $\lim _{n} d\left(f g x_{n}, g f x_{n}\right)=0$ with $\lim _{n} f x_{n}=\lim _{n} g x_{n}=t$ for some $t \in X$;

(vii) conditionally commuting [33] if they commute on a nonempty subset of the set of coincidence points whenever the set of their coincidence point is nonempty;

(viii) conditionally compatible [34] iff, whenever the set of sequences $\left\{x_{n}\right\}$ satisfying $\lim _{n} f x_{n}=\lim _{n} g x_{n}$ is nonempty, there exists a sequence $\left\{y_{n}\right\}$ such that $\lim _{n} f y_{n}=\lim _{n} g y_{n}=t$ (say) and $\lim _{n} d\left(f g y_{n}, g f y_{n}\right)=0$;

(ix) pseudo-compatible [40] iff, whenever the set of sequences $\left\{x_{n}\right\}$ satisfying $\lim _{n} f x_{n}=\lim _{n} g x_{n}$ is nonempty, there exists a sequence $\left\{y_{n}\right\}$ such that $\lim _{n} f y_{n}=\lim _{n} g y_{n}=t$ (say), $\lim _{n} d\left(f g y_{n}, g f y_{n}\right)=0$; and $\lim _{n} d\left(f g z_{n}, g f z_{n}\right)=0$ for any associated sequence $\left\{z_{n}\right\}$ of $\left\{y_{n}\right\}$.

We also recall that a pair $(f, g)$ of self-mappings defined on a metric space $(X, d)$ is said to be

(i) reciprocally continuous $[12,43]$ iff $\lim _{n} f g x_{n}=f t$ and $\lim _{n} g f x_{n}=g t$ whenever $\left\{x_{n}\right\}$ is a sequence in $X$ such that $\lim _{n} f x_{n}=\lim _{n} g x_{n}=t$ for some $t$ in $X$;

(ii) weakly reciprocally continuous [37] if $\lim _{n} f g x_{n}=f t$ or $\lim _{n} g f x_{n}=g t$ whenever $\left\{x_{n}\right\}$ is a sequence in $X$ such that $\lim _{n} f x_{n}=\lim _{n} g x_{n}=t$ for some $t$ in $X$;

(iii) conditionally reciprocally continuous (CRC) [38] if, whenever the set of sequences $\left\{x_{n}\right\}$ satisfying $\lim _{n} f x_{n}=\lim _{n} g x_{n}$ is nonempty, there exists a sequence $\left\{y_{n}\right\}$ satisfying $\lim _{n} f y_{n}=\lim _{n} g y_{n}=t$ (say) such that $\lim _{n} f g y_{n}=f t$ and $\lim _{n} g f y_{n}=g t$;

(iv) $g$-reciprocally continuous [40] iff $\lim _{n} f f x_{n}=f t$ and $\lim _{n} g f x_{n}=g t$ whenever $\left\{x_{n}\right\}$ is a sequence such that $\lim _{n} f x_{n}=\lim _{n} g x_{n}=t$ for some $t$ in $X$;

(v) sequentially continuous of type $\left(A_{g}\right)$ [39] iff there exists a sequence $\left\{x_{n}\right\}$ in $X$ such that $\lim _{n} f x_{n}=\lim _{n} g x_{n}=t$ for some $t \in X$ satisfying $\lim _{n} f f x_{n}=f t$ and $\lim _{n} g f x_{n}=g t$;

(vi) sequentially continuous of type $\left(A_{f}\right)$ [39] iff there exists a sequence $\left\{x_{n}\right\}$ in $X$ such that $\lim _{n} f x_{n}=\lim _{n} g x_{n}=t$ for some $t \in X$ satisfying $\lim _{n} f g x_{n}=f t$ and $\lim _{n} g g x_{n}=g t$ 
(vii) subsequentially continuous [21] iff there exists a sequence $x_{n}$ in $X$ such that $\lim _{n} f g x_{n}=f t$ and $\lim _{n} g f x_{n}=g t$ with $\lim _{n} f x_{n}=\lim _{n} g x_{n}=t$ for some $t \in X$.

Theorem 1.1 [40] Let $f$ and $g$ be g-reciprocally continuous self-mappings of a complete metric space $(X, d)$ such that

(i) $f X \subseteq g X$;

(ii) $d(f x, f y) \leq k d(g x, g y), k \in[0,1)$.

Iff and $g$ are pseudo-compatible, then $f$ and $g$ have a unique common fixed point.

Theorem 1.2 [40] Let $f$ and $g$ be g-reciprocally continuous noncompatible self-mappings of a metric space $(X, d)$ such that

(i) $f X \subseteq g X$;

(ii) $d(f x, f y)<\max \left\{d(g x, g y), \frac{k[d(f x, g x)+d(f y, g y)]}{2}, \frac{d(f x, g y)+d(f y, g x)}{2}\right\}$, where $1 \leq k<2$;

(iii) $d(x, f x) \neq \max \{d(x, g x), d(f x, g x)\}$,

whenever the right-hand side is nonzero. If $f$ and $g$ are pseudo-compatible, then $f$ and $g$ have a unique common fixed point.

Theorem 1.3 [34] Let $f$ and $g$ be conditionally compatible self-mappings of a metric space $(X, d)$ satisfying

$$
d(x, g x) \neq \max \{d(x, f x), d(g x, f x)\}
$$

whenever the right-hand side is nonzero. If $f$ and $g$ are noncompatible and reciprocally continuous, then $f$ and $g$ have a common fixed point.

Theorem 1.4 [38] Let $f$ and $g$ be conditionally reciprocal continuous self-mappings of a complete metric space $(X, d)$ such that

(i) $f X \subseteq g X$;

(ii) $d(f x, f y) \leq k d(g x, g y), k \in[0,1)$.

Iff and $g$ are either compatible or g-compatible or $f$-compatible, then $f$ and $g$ have a unique common fixed point.

Theorem 1.5 [44] Let $(X, d)$ be a complete metric space, let $f$ and $g$ be two noncompatible self-mappings on $X$ satisfying

$$
d(f x, f y) \leq \varphi(d(g x, g y)) \quad \text { for all } x, y \in X
$$

where $\varphi:[0, \infty) \rightarrow[0, \infty)$ is a continuous from right and nondecreasing function such that $\varphi(t)<t$ for all $t>0$. Assume that

(i) $\overline{f(X)} \subseteq g(X)$,

(ii) $\max \{d(g g x, f g x), d(f x, g f x)\} \leq \varphi(d(f x, g x))$ for all $x \in X$ and

(iii) $\varphi\left(d\left(f x, f^{2} x\right)\right) \neq \varphi\left(\max \left\{d(g x, g f x), d\left(g^{2} x, g f x\right), d(f x, g x), d\left(f^{2} x, g f x\right), d(f x, g f x)\right.\right.$, $\left.\left.d\left(g x, f^{2} x\right)\right\}\right)$, whenever $f x \neq f^{2} x$.

Then $f$ and $g$ have a unique common fixed point. Also, $f$ and $g$ are discontinuous at the common fixed point. 


\section{Main results}

We begin with the following example.

Example 2.1 Let $X=[2,20]$ and $d$ be the usual metric on $X$. Define self-mappings $f$ and $g$ on $X$ as follows:

$$
f x=\left\{\begin{array}{ll}
x+2 & \text { if } x \in(2,3], \\
5 & \text { otherwise, }
\end{array} \quad g x= \begin{cases}2 x & \text { if } x \in(2,3] \\
20 & \text { otherwise }\end{cases}\right.
$$

Then we can see that $f(X)=(4,5] \subseteq(4,6] \cup\{20\}=g(X)$ and the pair $(f, g)$ is $g$-reciprocally continuous. It can be verified that $d(f x, f y) \leq k d(g x, g y)$ for all $x, y \in X$ with $k=\frac{1}{2}$. Thus, $f$ and $g$ satisfy all the conditions of Theorem 1.1 except pseudo-compatibility. For the pseudo-compatibility, consider the only existent sequence $x_{n}=y_{n}=2+\frac{1}{n}$, then we have $\lim _{n} f y_{n}=\lim _{n} g y_{n}=4$, but $\lim _{n} f g y_{n}=\lim _{n} f\left(4+\frac{2}{n}\right)=5, \lim _{n} g f y_{n}=\lim _{n}\left(4+\frac{1}{n}\right)=20$, and so $\lim _{n} d\left(f g y_{n}, g f y_{n}\right)=15 \neq 0$. Also note that the pair $(f, g)$ is not compatible. Here, $(f, g)$ has no coincidence point therefore it is also not an occasionally weakly compatible but vacuously weakly compatible pair.

This suggests that pseudo-compatible is stronger than weakly compatible (and occasionally weakly compatible) in the context of Theorem 1.1 (such an observation is missing in [40]).

The above example motivated us to define the following.

Definition 2.1 Two self-mappings $f$ and $g$ of a metric space $(X, d)$ are called conditionally sequential absorbing if, whenever the set of sequences $\left\{x_{n}\right\}$ satisfying $\lim _{n} f x_{n}=\lim _{n} g x_{n}$ is nonempty, there exists a sequence $\left\{y_{n}\right\}$ satisfying $\lim _{n} f y_{n}=\lim _{n} g y_{n}=t$ (say) such that $\lim _{n} d\left(f y_{n}, f g y_{n}\right)=0$ and $\lim _{n} d\left(g y_{n}, g f y_{n}\right)=0$.

Example 2.2 Let $X=[2,10]$ and let $d$ be the usual metric on $X$. Define $f, g: X \rightarrow X$ as follows:

$$
f x=\left\{\begin{array}{ll}
2 & \text { if } x=2 \text { or } x>5, \\
6 & \text { if } x \in(2,5],
\end{array} \quad g x= \begin{cases}2 & \text { if } x=2, \\
2 x & \text { if } x \in(2,5] \\
\frac{x+1}{3} & \text { if } x>5 .\end{cases}\right.
$$

Then the maps are conditionally sequential absorbing. To view this, consider the constant sequence $x_{n}=2$. However, the pair $(f, g)$ is not weakly compatible as they do not commute at their coincidence point $x=3$. It may be noted that $x=2$ and $x=3$ are two coincidence points of $f$ and $g$. But in respect of the unique coincidence point (common fixed point), conditionally sequential absorbing always implies weakly compatible and hence occasionally weakly compatible and pseudo-compatible, because the maps naturally commute at their unique coincidence point (common fixed point).

Example 2.3 Let $X=[0,1]$ and let $d$ be the usual metric on $X$. Define $f, g: X \rightarrow X$ as follows:

$$
f x=1-x \text { and } g x=(1-x)^{2} \quad \text { for all } x \in X .
$$


Then $f$ and $g$ are weakly compatible but not conditionally sequential absorbing. Here, $x=0$ and $x=1$ are two coincidence points.

Remark 2.1 In Example 2.1, the pair $(f, g)$ is vacuously weakly compatible but not conditionally sequential absorbing and not pseudo-compatible. Note that $f$ and $g$ do not have any coincidence point. In Example 2.2, the pair $(f, g)$ is conditionally sequential absorbing but not weakly compatible. In Example 2.3, the pair $(f, g)$ is weakly compatible but not conditionally sequential absorbing.

Thus, as definitions, weakly compatible, pseudo-compatible and conditionally sequential absorbing are very different. However, in the context of a unique coincidence point, conditionally sequential absorbing is stronger than weakly compatible, which will be shown in our Example 2.6.

Example 2.4 Let $X=[0,+\infty)$ and let $d$ be the usual metric on $X$. Define $f, g: X \rightarrow X$ as follows:

$$
f x=\left\{\begin{array}{ll}
\frac{x}{3} & \text { if } x \in[0,1], \\
2 x-1 & \text { if } x>1,
\end{array} \quad g x= \begin{cases}\frac{x}{2} & \text { if } x \in[0,1] \\
3 x-2 & \text { if } x>1\end{cases}\right.
$$

Let us consider the sequence $x_{n}=\frac{1}{n}$ for $n=1,2, \ldots$ Then

$$
\begin{aligned}
& \lim _{n} f x_{n}=\lim _{n} \frac{1}{3 n}=0, \quad \lim _{n} g x_{n}=\lim _{n} \frac{1}{2 n}=0, \\
& \lim _{n} f g x_{n}=\lim _{n} \frac{1}{6 n}=0=f(0), \\
& \lim _{n} g f x_{n}=\lim _{n} \frac{1}{6 n}=0=g(0) .
\end{aligned}
$$

Thus $f$ and $g$ are conditionally reciprocal continuous and subsequentially continuous. We can see that $f$ and $g$ are neither weak reciprocal continuous nor $g$-reciprocal continuous. To see this, consider the sequence $x_{n}=1+\frac{1}{n}$ for $n=1,2, \ldots$, then

$$
\begin{aligned}
& \lim _{n} f x_{n}=\lim _{n}\left(1+\frac{2}{n}\right)=1, \quad \lim _{n} g x_{n}=\lim _{n}\left(1+\frac{3}{n}\right)=1, \\
& \lim _{n} f g x_{n}=\lim _{n}\left(1+\frac{6}{n}\right)=1 \neq f(1), \\
& \lim _{n} g f x_{n}=\lim _{n}\left(1+\frac{6}{n}\right)=1 \neq g(1), \\
& \lim _{n} f f x_{n}=\lim _{n}\left(1+\frac{4}{n}\right)=1 \neq f(1), \\
& \lim _{n} g g x_{n}=\lim _{n}\left(1+\frac{9}{n}\right)=1 \neq g(1) .
\end{aligned}
$$

Note that $f$ and $g$ do not have a coincidence point. 
Example 2.5 Let $X=R$ and let $d$ be the usual metric on $X$. Define $f, g: X \rightarrow X$ as follows:

$$
f x=x \text { and } g x=x+1 \quad \text { for all } x \in X .
$$

Then it is easy to see that the pair $(f, g)$ is reciprocal continuous, weak reciprocally continuous and conditionally reciprocally continuous but neither subsequentially continuous nor sequentially continuous of type $\left(A_{g}\right)$ and $\left(A_{f}\right)$. Note that the pair has no coincidence point.

In view of the above examples, we observe that in the event of no coincidence point, subsequential continuity as well as sequential continuity of type $\left(A_{g}\right)$ and $\left(A_{f}\right)$ are different from reciprocal continuity (respectively $g$-reciprocal and conditionally reciprocal continuity). However, in the context of a unique coincidence point (common fixed point), subsequential continuity as well as sequential continuity of type $\left(A_{g}\right)$ and $\left(A_{f}\right)$ are equivalent to conditionally reciprocal continuity.

The motivation of the following definition can be predicted from the proof of the last step in our Theorem 2.1.

Definition 2.2 Two self-mappings $f$ and $g$ of a metric space $(X, d)$ are called pseudoreciprocal continuous (PRC) (with respect to conditionally sequential absorbing) if, whenever the set of sequences $\left\{x_{n}\right\}$ satisfying $\lim _{n} f x_{n}=\lim _{n} g x_{n}$ is nonempty, there exists a sequence $\left\{y_{n}\right\}$ (satisfying $\lim _{n} f y_{n}=\lim _{n} g y_{n}=t$ (say), $\lim _{n} d\left(f y_{n}, f g y_{n}\right)=0$ and $\left.\lim _{n} d\left(g y_{n}, g f y_{n}\right)=0\right)$ such that $\lim _{n} f g y_{n}=f t$ and $\lim _{n} g f y_{n}=g t$.

\section{Common fixed point theorems}

Assume that $\phi, \psi:[0, \infty) \rightarrow[0, \infty)$ are two functions such that

(a) $\phi$ is nondecreasing, continuous and $\phi(0)=0<\phi(t)$ for every $t>0$;

(b) $\psi$ is nondecreasing, right-continuous, and $\psi(t)<t$ for every $t>0$.

To prove our first result, we use the following lemma.

Lemma 2.1 [45] For every function $\psi:[0, \infty) \rightarrow[0, \infty)$, let $\psi^{n}$ be the nth iterate of $\psi$. Then the following hold:

(i) if $\psi$ is nondecreasing, then for each $t>0, \lim _{n \rightarrow \infty} \psi^{n}(t)=0$ implies $\psi(t)<t$;

(ii) if $\psi$ is right-continuous with $\psi(t)<t$ for $t>0$, then $\lim _{n \rightarrow \infty} \psi^{n}(t)=0$.

Theorem 2.1 Let $f$ and $g$ be two pseudo-reciprocal continuous (w.r.t.conditionally sequential absorbing) self-mappings of a complete metric space $(X, d)$ such that $f X \subseteq g X$, and let $\phi, \psi:[0, \infty) \rightarrow[0, \infty)$ be two functions satisfying (a) and (b). If for all $x, y \in X$,

$$
\phi(d(f x, f y)) \leq \psi(\phi(M(x, y)))
$$

where

$$
M(x, y)=\max \left\{d(g x, g y), d(f x, g x), d(f y, g y), \frac{d(g x, f y)+d(f x, g y)}{2}\right\},
$$

then $f$ and $g$ have a unique common fixed point provided $(f, g)$ is conditionally sequential absorbing. 
Proof Let $x_{0} \in X$ and since $f X \subseteq g X$, so we have a sequence $\left\{p_{n}\right\}$ defined by

$$
p_{n}=f x_{n}=g x_{n+1} .
$$

Now we show that $\left\{p_{n}\right\}$ is a Cauchy sequence. We have

$$
\begin{aligned}
M\left(x_{n}, x_{n+1}\right)= & \max \left\{d\left(g x_{n}, g x_{n+1}\right), d\left(f x_{n}, g x_{n}\right), d\left(f x_{n+1}, g x_{n+1}\right),\right. \\
& \left.\frac{d\left(g x_{n}, f x_{n+1}\right)+d\left(f x_{n}, g x_{n+1}\right)}{2}\right\} \\
= & \max \left\{d\left(p_{n-1}, p_{n}\right), d\left(p_{n}, p_{n-1}\right), d\left(p_{n+1}, p_{n}\right),\right. \\
& \left.\frac{d\left(p_{n-1}, p_{n+1}\right)+d\left(p_{n}, p_{n}\right)}{2}\right\} \\
= & \max \left\{d\left(p_{n-1}, p_{n}\right), d\left(p_{n+1}, p_{n}\right), \frac{d\left(p_{n-1}, p_{n+1}\right)}{2}\right\} \\
= & \max \left\{d\left(p_{n-1}, p_{n}\right), d\left(p_{n+1}, p_{n}\right)\right\} .
\end{aligned}
$$

If we suppose $M\left(x_{n}, x_{n+1}\right)=d\left(p_{n}, p_{n+1}\right)$, then

$$
\begin{aligned}
\phi\left(d\left(f x_{n}, f x_{n+1}\right)\right) & \leq \psi\left(\phi\left(M\left(x_{n}, x_{n+1}\right)\right)\right)=\psi\left(\phi\left(d\left(p_{n}, p_{n+1}\right)\right)\right) \\
& =\psi\left(\phi\left(d\left(f x_{n}, f x_{n+1}\right)\right)\right)<\phi\left(d\left(f x_{n}, f x_{n+1}\right)\right),
\end{aligned}
$$

which is a contradiction. Therefore

$$
M\left(x_{n}, x_{n+1}\right)=d\left(p_{n-1}, p_{n}\right) .
$$

Similarly,

$$
M\left(x_{n}, x_{n-1}\right)=d\left(p_{n-1}, p_{n-2}\right) .
$$

If for some $n$ we have either $p_{n}=p_{n-1}$ or $p_{n}=p_{n+1}$, then by condition (2.1) we obtain that the sequence $\left\{p_{n}\right\}$ is definitely constant and thus it is a Cauchy sequence. Suppose $p_{n} \neq p_{n-1}$ for each $n$, then from condition (2.1) we have

$$
\begin{aligned}
\phi\left(d\left(p_{n}, p_{n-1}\right)\right) & =\phi\left(d\left(f x_{n}, f x_{n-1}\right)\right) \leq \psi\left(\phi\left(M\left(x_{n}, x_{n-1}\right)\right)\right) \\
& =\psi\left(\phi\left(d\left(p_{n-1}, p_{n-2}\right)\right)\right)<\phi\left(d\left(p_{n-1}, p_{n-2}\right)\right),
\end{aligned}
$$

and for all $n \in N$,

$$
\phi\left(d\left(p_{n}, p_{n+1}\right)\right)<\phi\left(d\left(p_{n-1}, p_{n}\right)\right) .
$$

Now, we have

$$
\phi\left(d\left(p_{n+1}, p_{n}\right)\right) \leq \psi\left(\phi\left(d\left(p_{n}, p_{n-1}\right)\right)\right) \leq \cdots \leq \psi^{n}\left(\phi\left(d\left(p_{0}, p_{1}\right)\right)\right)
$$


and then, by Lemma 2.1(ii),

$$
\begin{gathered}
\lim _{n \rightarrow \infty} \psi^{n}\left(\phi\left(d\left(p_{n+1}, p_{n}\right)\right)\right)=0 \\
\Rightarrow \lim _{n \rightarrow \infty} \phi\left(d\left(p_{n+1}, p_{n}\right)\right)=0 \\
\Rightarrow \lim _{n \rightarrow \infty} d\left(p_{n+1}, p_{n}\right)=0 .
\end{gathered}
$$

Now we prove that $\left\{p_{n}\right\}$ is Cauchy.

Suppose not, then $\exists \epsilon>0$ such that $d\left(p_{n}, p_{m}\right) \geq 2 \epsilon$ for infinite value of $m$ and $n$ with $m<n$. This assumes that there exist two sequences $\left\{m_{k}\right\},\left\{n_{k}\right\}$ of natural numbers with $m_{k}<n_{k}$ such that

$$
d\left(p_{m_{k}}, p_{n_{k}+1}\right)>\epsilon .
$$

It is not restrictive to suppose that $n_{k}$ is the least positive integer exceeding $m_{k}$ and satisfying (2.5). We have

$$
\begin{aligned}
\epsilon & <d\left(p_{m_{k}}, p_{n_{k}+1}\right) \\
& \leq d\left(p_{m_{k}}, p_{n_{k}-1}\right)+d\left(p_{n_{k}-1}, p_{n_{k}}\right)+d\left(p_{n_{k}}, p_{n_{k}+1}\right) \\
& \leq \epsilon+d\left(p_{n_{k}-1}, p_{n_{k}}\right)+d\left(p_{n_{k}}, p_{n_{k}+1}\right) .
\end{aligned}
$$

Then

$$
d\left(p_{m_{k}}, p_{n_{k}+1}\right) \rightarrow \epsilon \quad \text { as } k \rightarrow \infty .
$$

We note

$$
\begin{aligned}
& d\left(p_{m_{k}}, p_{n_{k}+1}\right)-d\left(p_{m_{k}}, p_{m_{k}+1}\right)-d\left(p_{n_{k}+2}, p_{n_{k}+1}\right) \\
& \quad \leq d\left(p_{m_{k}+1}, p_{n_{k}+2}\right) \leq d\left(p_{m_{k}}, p_{n_{k}+1}\right)-d\left(p_{m_{k}}, p_{m_{k}+1}\right)-d\left(p_{n_{k}+2}, p_{n_{k}+1}\right),
\end{aligned}
$$

and therefore

$$
d\left(p_{m_{k}+1}, p_{n_{k}+2}\right) \rightarrow \epsilon \quad \text { as } k \rightarrow \infty .
$$

Now, we have

$$
\begin{aligned}
M\left(x_{n_{k}+2}, x_{m_{k}+1}\right)= & \max \left\{d\left(p_{m_{k}}, p_{n_{k}+1}\right), d\left(p_{n_{k}+1}, p_{n_{k}+2}\right), d\left(p_{m_{k}}, p_{m_{k}+1}\right),\right. \\
& \left.\frac{d\left(p_{m_{k}+1}, p_{n_{k}+1}\right)+d\left(p_{m_{k}}, p_{n_{k}+2}\right)}{2}\right\} \\
= & d\left(p_{m_{k}}, p_{n_{k}+1}\right)+d_{k},
\end{aligned}
$$

where $d_{k} \rightarrow 0$ as $k \rightarrow+\infty$ and $d_{k} \geq 0$ for all $k$. Then from

$$
\begin{aligned}
\phi\left(d\left(p_{m_{k}+1}, p_{n_{k}+2}\right)\right) & =\phi\left(d\left(f x_{n_{k}+2}, f x_{m_{k}+1}\right)\right) \leq \psi\left(\phi\left(M\left(x_{n_{k}+2}, x_{m_{k}+1}\right)\right)\right) \\
& =\psi\left(\phi\left(d\left(p_{m_{k}}, p_{n_{k}+1}\right)+d_{k}\right)\right),
\end{aligned}
$$


as $k \rightarrow+\infty, \phi$ being continuous and $\psi$ right-continuous, we get

$$
\phi(\epsilon) \leq \psi(\phi(\epsilon))<\phi(\epsilon) .
$$

This is a contradiction. Therefore $\left\{p_{n}\right\}$ is a Cauchy sequence. Since $(X, d)$ is a complete metric space, therefore $\exists t \in X$ such that

$$
p_{n}=f x_{n}=g x_{n+1} \rightarrow t \quad \text { as } n \rightarrow \infty .
$$

Since the pair $(f, g)$ is conditionally sequential absorbing, therefore there exists a sequence $\left\{y_{n}\right\}$ in $X$ such that $f y_{n} \rightarrow u, g y_{n} \rightarrow u$ (say) satisfying

$$
\lim _{n} d\left(f y_{n}, f g y_{n}\right)=0 \text { and } \lim _{n} d\left(g y_{n}, g f y_{n}\right)=0,
$$

and by pseudo-reciprocal continuity (w.r.t. conditionally sequential absorbing) of $(f, g)$, we have

$$
\lim _{n} f g y_{n}=f u \text { and } \lim _{n} g f y_{n}=g u .
$$

In view of (2.11) and (2.12), we get $f u=g u=u$, i.e., $u$ is a common fixed point of $f$ and $g$. The uniqueness of a common fixed point follows easily from contractive condition (2.1).

Example 2.6 Let $X=[0,1]$ with the usual metric $d$. Define self-maps $f$ and $g$ as follows:

$$
f x=\left\{\begin{array}{ll}
\frac{1+x}{2} & \text { if } x \in\left[0, \frac{1}{2}\right), \\
\frac{1}{2} & \text { if } x \in\left[\frac{1}{2}, 1\right],
\end{array} \quad g x= \begin{cases}\frac{1}{2}+x & \text { if } x \in\left[0, \frac{1}{2}\right), \\
\frac{1}{2} & \text { if } x=\frac{1}{2}, \\
\frac{4}{5} & \text { if } x \in\left(\frac{1}{2}, 1\right] .\end{cases}\right.
$$

Then $f$ and $g$ satisfy all the conditions of Theorem 2.1 with $f(X)=\left[\frac{1}{2}, \frac{3}{4}\right) \subseteq\left[\frac{1}{2}, 1\right)=g(X)$. Here, $f$ and $g$ are conditionally sequential absorbing and pseudo-reciprocal continuous (w.r.t. conditionally sequential absorbing) in respect of the constant sequence $x_{n}=\frac{1}{2}$. Let us consider the sequence $x_{n}=\frac{1}{n+2}$, then

$$
\begin{aligned}
& \lim _{n} f x_{n}=\lim _{n}\left(\frac{1}{2}+\frac{1}{2 n+4}\right)=\frac{1}{2}=\lim _{n}\left(\frac{1}{2}+\frac{1}{n+2}\right)=\lim _{n} g x_{n}, \\
& \lim _{n} f g x_{n}=\lim _{n} f\left(\frac{1}{2}+\frac{1}{n+2}\right)=\frac{1}{2}=f\left(\frac{1}{2}\right), \\
& \lim _{n} g f x_{n}=\lim _{n} g\left(\frac{1}{2}+\frac{1}{2 n+4}\right)=\frac{4}{5} \neq g\left(\frac{1}{2}\right), \\
& \lim _{n} f f x_{n}=\lim _{n} f\left(\frac{1}{2}+\frac{1}{2 n+4}\right)=\frac{1}{2}=f\left(\frac{1}{2}\right), \\
& \lim _{n} g g x_{n}=\lim _{n} g\left(\frac{1}{2}+\frac{1}{n+2}\right)=\frac{4}{5} \neq g\left(\frac{1}{2}\right) .
\end{aligned}
$$

Thus, $(f, g)$ is not a reciprocal as well as not a $g$-reciprocal continuous pair. Also the pair $(f, g)$ is neither compatible, $f$-compatible nor $g$-compatible. 
If we take $\phi(t)=t$ and $\psi(t)=k t, k \in[0,1)$, then it can be verified that $f$ and $g$ satisfy contraction condition (2.1) with $k=\frac{5}{6}$. Here, $x=\frac{1}{2}$ is the unique common fixed point of $f$ and $g$, which is also a point of discontinuity.

On the other hand, notice that at $x=1, f$ and $g$ do not satisfy the condition

$$
\max \{d(g g x, f g x), d(f f x, g f x)\} \leq \varphi(d(f x, g x))
$$

used in Theorem 1.5. Here, it is worth noting that none of the earlier relevant theorems, for example, Theorem 1.1, Theorem 1.4 and Theorem 1.5, can be used in the context of this example. One more interesting part of this example is that neither $f(X)$ nor $g(X)$ is closed. Thus the result of Singh and Mishra [19] cannot be applicable in the context of this example.

Theorem 2.2 Let $f$ and $g$ be pseudo-reciprocal continuous (w.r.t. conditionally sequential absorbing) and noncompatible self-mappings of a metric space $(X, d)$ satisfying

$$
d(f x, f y)<\max \left\{d(g x, g y), \frac{k[d(f x, g x)+d(f y, g y)]}{2}, \frac{d(f x, g y)+d(f y, g x)}{2}\right\},
$$

where $1 \leq k<2$. If $f$ and $g$ are conditionally sequential absorbing, then $f$ and $g$ have a unique common fixed point.

Proof Since $f$ and $g$ are noncompatible maps, there exists a sequence $\left\{x_{n}\right\}$ in $X$ such that $f x_{n} \rightarrow t$ and $g x_{n} \rightarrow t$ for some $t$ in $X$ but either $\lim _{n} d\left(f g x_{n}, g f x_{n}\right) \neq 0$ or the limit does not exist. Also, the pair $(f, g)$ is conditionally sequential absorbing; therefore, there exists a sequence $\left\{y_{n}\right\}$ in $X$ such that $\lim _{n} f y_{n}=\lim _{n} g y_{n}=u$ (say) with $\lim _{n} d\left(f y_{n}, f g y_{n}\right)=0$ and $\lim _{n} d\left(g y_{n}, g f y_{n}\right)=0$. Now, by the pseudo-reciprocal continuity (w.r.t. conditionally sequential absorbing) of the pair $(f, g)$, we have $f g y_{n} \rightarrow f u$ and $g f y_{n} \rightarrow g u$. In view of these limits, we get $u$ is a common fixed point of $f$ and $g$.

Now, suppose that there exists another common fixed point $w$ of $f$ and $g$ such that $w \neq u$. Then, on using (2.13), we have

$$
d(f w, f u)<\max \left\{d(g w, g u), \frac{k[d(f w, g w)+d(f u, g u)]}{2}, \frac{d(f w, g u)+d(f u, g w)}{2}\right\} .
$$

Thus, we have $d(w, u)<\frac{k}{2} d(w, u)<d(w, u)$, a contradiction and hence $w=u$.

Example 2.7 Again consider Example 2.6 wherein the pair $(f, g)$ satisfies all the conditions of Theorem 2.2 for all $k \in[1,2)$. Note that at $x=0, f$ and $g$ do not satisfy the condition

$$
d(x, f x) \neq \max \{d(x, g x), d(f x, g x)\}
$$

whenever the right-hand side is nonzero. Thus Theorem 2.2 is a genuine extension and improvement of Theorem 1.2 due to Pant and Bisht [40].

Observation The proof of Theorem 2.1, Theorem 2.2 and examples above immediately suggest us defining another type of continuity as follows. 
Definition 2.3 Two self-mappings $f$ and $g$ of a metric space $(X, d)$ are called pseudoreciprocal continuous (PRC) (with respect to pseudo-compatible) if whenever the set of sequences $\left\{x_{n}\right\}$ satisfying $\lim _{n} f x_{n}=\lim _{n} g x_{n}$ is nonempty, there exists a sequence $\left\{y_{n}\right\}$ (satisfying $\lim _{n} f y_{n}=\lim _{n} g y_{n}=t$ (say); $\lim _{n} d\left(f g y_{n}, g f y_{n}\right)=0$; and $\lim _{n} d\left(f g z_{n}, g f z_{n}\right)=0$ for any associated sequence $\left\{z_{n}\right\}$ of $\left.\left\{y_{n}\right\}\right)$ such that $\lim _{n} f g y_{n}=f t$ and $\lim _{n} g f y_{n}=g t$.

However, the notions of pseudo-compatibility and pseudo-reciprocal continuity (w.r.t. pseudo-compatibility) are no more applicable in the context of the existence of nonunique common fixed points for a pair of maps. This fact is illustrated in Example 2.11 below. At the same time, conditionally sequential absorbing and pseudo-reciprocal continuity (w.r.t. conditionally sequential absorbing) are easily applicable.

Theorem 2.3 Let $f$ and $g$ be reciprocal (or g-reciprocal) continuous and noncompatible self-mappings of a metric space $(X, d)$ satisfying (2.13). Then the pair $(f, g)$ has a unique common fixed point provided it is conditionally sequential absorbing. Moreover, $f$ and $g$ are discontinuous at the common fixed point.

Proof Since $f$ and $g$ are noncompatible, there exists a sequence $\left\{x_{n}\right\}$ in $X$ such that $\lim _{n} f x_{n}=\lim _{n} g x_{n}=t$ for some $t \in X$, but $\lim _{n} d\left(f g x_{n}, g f x_{n}\right)$ is either nonzero or not existent. Also, since $f$ and $g$ are conditionally sequential absorbing and $\lim _{n} f x_{n}=\lim _{n} g x_{n}=$ $t$, there exists a sequence $\left\{y_{n}\right\}$ in $X$, satisfying $\lim _{n} f y_{n}=\lim _{n} g y_{n}=u$ (say), such that $\lim _{n} d\left(f y_{n}, f g y_{n}\right)=0$ and $\lim _{n} d\left(g y_{n}, g f y_{n}\right)=0$. The reciprocal continuity of the pair $(f, g)$ implies that $\lim _{n} f g y_{n}=f u$ and $\lim _{n} g f y_{n}=g u$. Thus, in view of these limits, we obtain $f u=$ $g u=u$. If we consider the pair $(f, g) g$-reciprocal continuous, then we have $\lim _{n} f f y_{n}=f u$ and $\lim _{n} g f y_{n}=g u$. Since $\lim _{n} d\left(g y_{n}, g f y_{n}\right)=0$, so we have $g u=u$. Now, suppose $f u \neq u$. On using (2.13), we get $d(f u, u)<\frac{k}{2} d(f u, u)<d(f u, u)$, a contradiction and hence $f u=u$. Thus $u$ is a common fixed point of $f$ and $g$. Applying (2.13), we can show the uniqueness of the common fixed point.

We now show that $f$ and $g$ are discontinuous at the common fixed point $u$. If possible, suppose $f$ is continuous at $u$. Then, considering the sequence $\left\{x_{n}\right\}$ of the present theorem and on using (2.13), we get $t=u$ and hence by the continuity of $f$, we have $f f x_{n} \rightarrow f u=u$ and $f g x_{n} \rightarrow f u=u$. Now, reciprocal (or $g$-reciprocal) continuity of the pair $(f, g)$ implies that $g f x_{n} \rightarrow g u=u$. This further yields that $\lim _{n} d\left(f g x_{n}, g f x_{n}\right)=0$, which contradicts the fact that $\lim _{n} d\left(f g x_{n}, g f x_{n}\right)$ is either nonzero or non-existent. Hence $f$ is discontinuous at the fixed point.

Next, suppose that $g$ is continuous at $u$. Then, for the sequence $\left\{x_{n}\right\}$, we get $g f x_{n} \rightarrow$ $g u=u$ and $g g x_{n} \rightarrow g u=u$. If $(f, g)$ is reciprocal continuous, then we have $f g x_{n} \rightarrow f u=u$, and if it is $g$-reciprocal continuous, then on using (2.13), we get $f g x_{n} \rightarrow f u=u$. Thus, we obtain $\lim _{n} d\left(f g x_{n}, g f x_{n}\right)=0$, a contradiction. Therefore $f$ and $g$ are discontinuous at their common fixed point.

Example 2.8 Let $X=[2,20]$ with the usual metric $d$. Define $f, g: X \rightarrow X$ as follows:

$$
f x=\left\{\begin{array}{ll}
2 & \text { if } x=2, x>5, \\
4 & \text { if } 2<x \leq 5,
\end{array} \quad g x= \begin{cases}2 & \text { if } x=2, x \geq 8 \\
20 & \text { if } 2<x \leq 5 \\
\frac{x+1}{3} & \text { if } 5<x<8\end{cases}\right.
$$


Then $f$ and $g$ satisfy all the conditions of Theorem 2.3. It can be verified in this example that $f$ and $g$ satisfy contractive condition (2.13) for all $k \in[1,2)$. To see that $f$ and $g$ are noncompatible, consider the sequence $\left\{x_{n}\right\}$ in $X$ such that $x_{n}=5+\epsilon_{n}$, then $f x_{n} \rightarrow 2, g x_{n}=$ $\left(2+\frac{\epsilon_{n}}{3}\right) \rightarrow 2, f g x_{n} \rightarrow 4, g f x_{n} \rightarrow 2$, and so $\lim _{n} d\left(f g x_{n}, g f x_{n}\right) \neq 0$. Also here the pair $(f, g)$ is $g$-reciprocal continuous. To see this, let $\left\{x_{n}\right\}$ be a sequence in $X$ such that $\lim _{n} f x_{n}=$ $\lim _{n} g x_{n}=t$ for some $t$ in $X$. Then $t=2, x_{n}=2$ or $x_{n}=5+\epsilon_{n}, f f x_{n} \rightarrow 2=f(2)$ and $g f x_{n} \rightarrow$ $2=g(2)$. The pair $(f, g)$ is conditionally sequential absorbing in respect of the constant sequence $\left\{y_{n}\right\}$ given by $y_{n}=2$. Here, $x=2$ is the unique common fixed point where $f$ and $g$ are discontinuous.

Note that at $x=8, f$ and $g$ do not satisfy the condition

$$
d(x, f x) \neq \max \{d(x, g x), d(f x, g x)\}
$$

whenever the right-hand side is nonzero. Also notice that at $x=3, f$ and $g$ do not satisfy

$$
\max \{d(g g x, f g x), d(f f x, g f x)\} \leq \varphi(d(f x, g x)) .
$$

Thus, Theorem 2.3 is a genuine extension and improvement of Theorem 1.2 due to Pant and Bisht [40] and Theorem 1.5 due to Rezapour and Shahzad [44].

In the absence of contractive condition (2.13), the following corollaries are straightforward from Theorems 2.2 and 2.3.

Corollary 2.1 Let $f$ and $g$ be pseudo-reciprocal continuous (w.r.t.conditionally sequential absorbing) and noncompatible self-mappings of a metric space $(X, d)$. Then $f$ and $g$ have a common fixed point provided it is conditionally sequential absorbing.

Corollary 2.2 Let $f$ and $g$ be reciprocal (or g-reciprocal) continuous and noncompatible self-mappings of a metric space $(X, d)$. Then the pair $(f, g)$ has a common fixed point provided it is conditionally sequential absorbing.

The following examples illustrate the above corollaries.

Example 2.9 Consider $X=[2,23]$ and let $d$ be the usual metric on $X$. Define $f, g: X \rightarrow X$ as

$$
f x= \begin{cases}2 & \text { if } x \in\{2\} \cup(5,7) \cup(7,10) \cup(10,11) \cup(11,12) \\ & \cup(12,13) \cup(13,21) \cup(21,23) \\ \frac{x+5}{2} & \text { if } 2<x \leq 5 \\ 7 & \text { if } x=7,23 \\ 12 & \text { if } x=10 \\ 11 & \text { if } x=11,13 \\ 11.5 & \text { if } x=12 \\ 10 & \text { if } x=21\end{cases}
$$




$$
g x= \begin{cases}2 & \text { if } x \in\{2\} \cup[7,10) \cup(10,11) \cup(11,12) \cup(12,13) \\ & \cup(13,21) \cup(21,22) \cup(22,23) \\ 6 & \text { if } 2<x \leq 5 \\ \frac{x+1}{3} & \text { if } x \in(5,7) \\ 11 & \text { if } x=10,11,13,22 \\ 11.6 & \text { if } x=12 \\ 10 & \text { if } x=21 \\ 7 & \text { if } x=23 .\end{cases}
$$

Here $f$ and $g$ satisfy all the conditions of Corollary 2.1. In view of the constant sequence $x_{n}=2$ or $x_{n}=11$, the pair $(f, g)$ is conditionally sequential absorbing and pseudoreciprocal continuous (w.r.t. conditionally sequential absorbing). For noncompatibility as well as non-reciprocal continuity, let us consider the sequence $x_{n}=5+\left(\frac{1}{n}\right)$, then we have

$$
\begin{aligned}
& f x_{n} \rightarrow 2, \quad g x_{n}=\left(2+\frac{1}{3 n}\right) \rightarrow 2 \\
& \lim _{n} f g x_{n}=\lim _{n}\left(\frac{7}{2}+\frac{1}{6 n}\right)=\frac{7}{2} \neq f(2), \\
& \lim _{n} g f x_{n}=2=g(2),
\end{aligned}
$$

and so $\lim _{n} d\left(f g x_{n}, g f x_{n}\right) \neq 0$. Here, 2 and 11 are two common fixed points of $f$ and $g$. Also the pair is not weakly compatible as $f$ and $g$ do not commute at their coincidence point $x=23$.

Note that at $x=13$ and $y=22$ the present example does not satisfy condition (2.13) for any $k \in[1,2)$ and also Lipschitz-type condition used in [33] for any $k \geq 0$. Also notice that at $x=21$, the involved maps do not satisfy any of the conditions:

(i) $d(x, f x) \neq \max \{d(x, g x), d(f x, g x)\}$,

(ii) $d(x, g x) \neq \max \{d(x, g x), d(g x, f x)\}$,

(iii) $d(x, g x) \neq \max \{d(x, f x), d(g x, f x)\}$, and

(iv) $d\left(f x, f^{2} x\right) \neq \max \left\{d(g x, g f x), d(f x, g x), d\left(f^{2} x, g f x\right), d(f x, g f x), d\left(g x, f^{2} x\right)\right\}$,

whenever the right-hand side is nonzero. Here, it is worth noting that none of the Theorem 1.3 due to Pant and Bisht [34] and the main results contained in Pant and Pant [33] and Gopal et al. [46] can be used in the context of Corollary 2.1.

Example 2.10 Consider $X=[2,23]$ and let $d$ be the usual metric on $X$. Define $f, g: X \rightarrow X$ as

$$
f x= \begin{cases}2 & \text { if } x \in[2,7) \cup(7,10) \cup(10,11) \cup(11,12) \\ & \cup(12,13) \cup(13,21) \cup(21,23), \\ 7 & \text { if } x=7,23 \\ 12 & \text { if } x=10, \\ 11 & \text { if } x=11,13, \\ 11.5 & \text { if } x=12, \\ 10 & \text { if } x=21,\end{cases}
$$




$$
g x= \begin{cases}2 & \text { if } x \in\{2\} \cup(5,10) \cup(10,11) \cup(11,12) \cup(12,13) \\ 6 & \text { if } x \in(2,5] \\ 11 & \text { if } x=10,11,13,22 \\ 11.6 & \text { if } x=12 \\ 10 & \text { if } x=21 \\ 7 & \text { if } x=23\end{cases}
$$

In this example the pair $(f, g)$ is noncompatible as well as reciprocal continuous and satisfies all the conditions of Corollary 2.2. Let us consider the sequence $x_{n}=23$, then $f x_{n} \rightarrow 7, g x_{n} \rightarrow 7$ and

$$
\begin{aligned}
& f g x_{n} \rightarrow 7=f(7), \\
& g f x_{n} \rightarrow 2=g(7),
\end{aligned}
$$

therefore $\lim _{n} d\left(f g x_{n}, g f x_{n}\right) \neq 0$, and so $(f, g)$ is noncompatible. Here, 2 and 11 are two common fixed points of $f$ and $g$.

Finally, we present an example which shows that the requirement of conditionally sequential absorbing property is necessary for producing common fixed points of mappings satisfying non-expansive or Lipschitz-type conditions besides exhibiting the limitations of commuting properties of the pairs utilized in earlier related results of Pant and Bisht [34], Pant and Pant [33] and Jungck and Rhoades [17].

Example 2.11 Let $X=[2,20]$ endowed with the usual metric $d$ and $f, g: X \rightarrow X$ by

$$
f x=\left\{\begin{array}{ll}
6 & \text { if } 2 \leq x<6 \text { or } x>6, \\
\frac{13}{2} & \text { if } x=6,
\end{array} \quad g x= \begin{cases}5 & \text { if } 2 \leq x \leq 5, \\
\frac{x+7}{2} & \text { if } 5<x \leq 6 \\
10 & \text { if } 6<x<13 / 2 \text { or } x>13 / 2 \\
6 & \text { if } x=13 / 2 .\end{cases}\right.
$$

Then by a routine calculation, it can be verified that $\overline{f(X)} \subseteq g(X)$ and $d(f x, f y) \leq k d(g x, g y)$ for all $x, y \in X$, where $k \geq 0$. Also, $f$ and $g$ are a noncompatible and weakly commuting (and hence occasionally weakly compatible and conditionally commuting) pair. In order to show that $(f, g)$ is noncompatible, the sequence $x_{n}=5+1 / n ; n>1, n \in N$ satisfies the requirements. Also, it is straightforward to verify that the pair $(f, g)$ is pseudo-compatible as well as pseudo-reciprocal continuous (w.r.t. pseudo-compatible), but the pair is not conditionally sequential absorbing in respect of $x_{n}=6$ or $5+1 / n$. On the other hand, at $x=6$, it can be verified that the mappings $f$ and $g$ do not satisfy any one of the conditions described by (i), (ii), (iii) or (iv) mentioned earlier. Notice that the estimated pair has no common fixed point.

Observations The following definitions can be considered as variants of conditionally sequential absorbing. Two self-mappings $f$ and $g$ of a metric space $(X, d)$ are called conditionally sequential absorbing 
(i) of type (A) if, whenever the set of sequences $\left\{x_{n}\right\}$ satisfying $\lim _{n} f x_{n}=\lim _{n} g x_{n}$ is nonempty, there exists a sequence $\left\{y_{n}\right\}$ satisfying $\lim _{n} f y_{n}=\lim _{n} g y_{n}=t$ (say) such that $\lim _{n} d\left(f y_{n}, f f y_{n}\right)=0$ and $\lim _{n} d\left(g y_{n}, g f y_{n}\right)=0$;

(ii) of type (B) if, whenever the set of sequences $\left\{x_{n}\right\}$ satisfying $\lim _{n} f x_{n}=\lim _{n} g x_{n}$ is nonempty, there exists a sequence $\left\{y_{n}\right\}$ satisfying $\lim _{n} f y_{n}=\lim _{n} g y_{n}=t$ (say) such that $\lim _{n} d\left(f y_{n}, f g y_{n}\right)=0$ and $\lim _{n} d\left(g y_{n}, g g y_{n}\right)=0$;

(iii) of type (C) if, whenever the set of sequences $\left\{x_{n}\right\}$ satisfying $\lim _{n} f x_{n}=\lim _{n} g x_{n}$ is nonempty, there exists a sequence $\left\{y_{n}\right\}$ satisfying $\lim _{n} f y_{n}=\lim _{n} g y_{n}=t$ (say) such that $\lim _{n} d\left(f y_{n}, f y_{n}\right)=0$ and $\lim _{n} d\left(g y_{n}, g g y_{n}\right)=0$.

We can have some more variants by interchanging the place of $f$ and $g$. In respect of these variants, we can also define the corresponding pseudo-reciprocal continuity, for example, two self-mappings $f$ and $g$ of a metric space $(X, d)$ are called pseudo-reciprocal continuous of type (A) if, whenever the set of sequences $\left\{x_{n}\right\}$ satisfying $\lim _{n} f x_{n}=\lim _{n} g x_{n}$ is nonempty, there exists a sequence $\left\{y_{n}\right\}$ (satisfying $\lim _{n} f y_{n}=\lim _{n} g y_{n}=t\left(\right.$ say), $\lim _{n} d\left(f y_{n}, f f y_{n}\right)=0$ and $\left.\lim _{n} d\left(g y_{n}, g f y_{n}\right)=0\right)$ such that $\lim _{n} f f y_{n}=$ ft and $\lim _{n} g f y_{n}=g t$.

Remark 2.2 The conclusion of our previous results will remain true if we replace the conditionally sequential absorbing and pseudo-reciprocal continuity by any one of the above variants of conditionally sequential absorbing and corresponding pseudo-reciprocal continuity. However, in the context of a unique coincidence or common fixed point, all these variants coincide with each others.

Competing interests

The authors declare that they have no competing interests.

Authors' contributions

All authors contributed equally and significantly in writing this paper. All authors read and approved the final manuscript.

\section{Author details}

${ }^{1}$ Department of Applied Mathematics \& Humanities, S. V. National Institute of Technology, Surat, Gujarat 395007, India.

2Department of Mathematics, Faculty of Science, King Mongkut's University of Technology Thonburi (KMUTT), 126 Pracha Uthit Rd., Bang Mod, Thung Khru, Bangkok, 10140, Thailand.

\section{Acknowledgements}

The second author thanks for the support of the King Mongkut's University of Technology Thonburi (KMUTT) and the third author is supported by CSIR, Govt. of India, grant number 25(0215)/13/EMR-II.

Received: 8 May 2013 Accepted: 26 June 2013 Published: 16 July 2013

\section{References}

1. Banach, S: Sur les operations dans les ensembles abstraits et leur applications aux equations intégrales. Fundam. Math. 3, 133-181 (1922)

2. Caccioppoli, R: Un teorema generale sull'esistenza di elementi uniti in una trasformazione funzionale. Rend. Lincei Mat. Appl. 11, 794-799 (1930) (in Italian)

3. Edelstein, M: On fixed points and periodic points under contractive mappings. J. Lond. Math. Soc. 1, 74-79 (1962)

4. Jungck, G: Commuting mappings and fixed points. Am. Math. Mon. 73, 261-263 (1976)

5. Boyce, WM: Commuting functions with no common fixed point. Trans. Am. Math. Soc. 137, 77-92 (1969)

6. Huneke, JP: On common fixed points of commuting continuous functions on an interval. Trans. Am. Math. Soc. 139, 371-381 (1969)

7. Murthy, PP: Important tools and possible applications of metric fixed point theory. Nonlinear Anal. 47, 3479-3490 (2001)

8. Chauhan, S, Sintunavarat, W, Kumam, P: Common fixed point theorems for weakly compatible mappings in fuzzy metric spaces using (JCLR) property. Appl. Math. 3(9), 976-982 (2012)

9. Jungck, G: Compatible mappings and common fixed points. Int. J. Math. Math. Sci. 9(4), 771-779 (1986)

10. Karapinar, E, Patel, DK, Imdad, M, Gopal, D: Some nonunique common fixed point theorems in symmetric spaces through CLR(S,T) property. Int. J. Math. Math. Sci. 2013, Article ID 753965 (2013)

11. Pant, RP: Common fixed points of noncommuting mappings. J. Math. Anal. Appl. 188, 436-440 (1994)

12. Pant, RP: Common fixed points of four mappings. Bull. Calcutta Math. Soc. 90, 281-286 (1998) 
13. Pathak, HK, Cho, YJ, Kang, SM: Remarks on R-weakly commuting mappings and common fixed point theorems. Bull. Korean Math. Soc. 34(2), 247-257 (1997)

14. Sessa, S: On a weak commutative condition of maps in fixed point consideration. Publ. Inst. Math. (Belgr.) 32(46), 149-153 (1982)

15. Sintunavarat, W, Kumam, P: Common fixed point theorems for a pair of weakly compatible mappings in fuzzy metric spaces. J. Appl. Math. 2011, Article ID 637958 (2011)

16. Sintunavarat, $W$, Kumam, $P$ : Common fixed points for $R$-weakly commuting in fuzzy metric spaces. Ann. Univ. Ferrara 58, 389-406 (2012)

17. Jungck, G, Rhoades, BE: Fixed point theorems for occasionally weakly compatible mappings. Fixed Point Theory $7(2)$, 287-296 (2006) (Erratum: Fixed Point Theory 9, 383-384 (2008))

18. Al-Thagafi, MA, Shahzad, N: Generalized /-nonexpansive selfmaps and invariant approximations. Acta Math. Sin. 24 867-876 (2008)

19. Singh, SL, Mishra, SN: Remarks on Jachymski's fixed point theorems for compatible maps. Indian J. Pure Appl. Math. 28, 611-645 (1997)

20. Suzuki, T, Pathak, HK: Almost biased mappings and almost compatible mappings are equivalent under some condition. J. Math. Anal. Appl. 368, 211-217 (2010)

21. Bouhadjera, H, Godet-Thobie, C: Common fixed point theorems for pair of subcompatible maps. arXiv:0906.3159v2 [math.FA]

22. Hussain, N, Khamsi, MA, Latif, A: Common fixed points for JH-operators and occasionally weakly biased pairs under relaxed conditions. Nonlinear Anal. 74, 2133-2140 (2011)

23. Sintunavarat, W, Kumam, P: Common fixed point theorems for generalized $\mathcal{J} \mathcal{H}$-operator classes and invariant approximations. J. Inequal. Appl. 2011, 67 (2011)

24. Dorić, D, Kadelburg, Z, Radenović, S: A note on occasionally weakly compatible mappings and common fixed points. Fixed Point Theory 13(2), 475-480 (2012)

25. Alghamdi, MA, Radenović, S, Shahzad, N: On some generalizations of commuting mappings. Abstr. Appl. Anal. 2012, Article ID 952052 (2012)

26. Babu, GVR, Alemayehu, GM: Common fixed point theorems for occasionally weakly compatible maps satisfying property (E. A) using an inequality involving quadratic terms. Appl. Math. Lett. 24, 975-981 (2011)

27. Bhatt, A, Chandra, H, Sahu, DR: Common fixed point theorems for occasionally weakly compatible mappings under relaxed conditions. Nonlinear Anal. 73, 176-182 (2010)

28. Ćirić, L, Samet, B, Vetro, C: Common fixed point theorems for families of occasionally weakly compatible mappings. Math. Comput. Model. 53, 631-636 (2011)

29. Jain, MK, Rhoades, BE, Saluja, AS: Fixed point theorems for occasionally weakly compatible expansive mappings. J. Adv. Math. Stud. 5(2), 54-58 (2012)

30. Sintunavarat, W, Petruşel, A, Kumam, P: Coupled common fixed point theorems for $W^{*}$-compatible mappings without mixed monotone property. Rend. Circ. Mat. Palermo 61, 361-383 (2012)

31. Abbas, M, Gopal, D, Radenović, S: A note on recently introduced commutative conditions. Indian J. Math. (2011, accepted)

32. Imdad, M, Ali, J, Tanveer, M: Remarks on some recent metrical common fixed point theorems. Appl. Math. Lett. 24(7), 1165-1169 (2011)

33. Pant, V, Pant, RP: Common fixed points of conditionally commuting maps. Fixed Point Theory 11(1), 113-118 (2010)

34. Pant, RP, Bisht, RK: Occasionally weakly compatible mappings and fixed points. Bull. Belg. Math. Soc. Simon Stevin 19, 1-7 (2012)

35. Pant, RP: Discontinuity and fixed points. J. Math. Anal. Appl. 240, 284-289 (1999)

36. Kannan, R: Some results on fixed points. Bull. Calcutta Math. Soc. 60, 71-76 (1968)

37. Pant, RP, Bisht, RK, Arora, D: Weak reciprocal continuity and fixed point theorems. Ann. Univ. Ferrara 57(1), 181-190 (2011)

38. Pant, RP, Bisht, RK: Common fixed point theorems under a new continuity condition. Ann. Univ. Ferrara (2011) doi:10.1007/s11565-011-0141-5

39. Gopal, D, Imdad, M, Abbas, M: Metrical common fixed point theorems without completeness and closedness. Fixed Point Theory Appl. 2012, 18 (2012)

40. Pant, RP, Bisht, RK: Common fixed points of pseudo compatible mappings. Rev. R. Acad. Cienc. Exactas Fís. Nat., Ser. A Mat. (2013). doi:10.1007/s13398-013-0119-5

41. Pathak, HK, Khan, MS: A comparison of various types of compatible maps and common fixed points. Indian J. Pure Appl. Math. 28(4), 477-485 (1997)

42. Pant, RP: Common fixed points of Lipschitz type mapping pairs. J. Math. Anal. Appl. 240, 280-283 (1999)

43. Pant, RP: A common fixed point theorem under a new condition. Indian J. Pure Appl. Math. 30(2), 147-152 (1999)

44. Rezapour, SH, Shahzad, N: Common fixed point of $(\psi, \phi)$-type contractive maps. Appl. Math. Lett. (2011). doi:10.1016/j.aml.2011.11.004

45. Matkowski, J: Fixed point theorems for mappings with a contractive iterate at a point. Proc. Am. Math. Soc. 62(2), 344-348 (1977)

46. Gopal, D, Imdad, M, Hasan, M, Patel, DK: Proving common fixed point theorems for Lipschitz type mappings via absorbing pair. Bull. Math. Anal. Appl. 3(4), 92-100 (2011) (Erratum: Bull. Math. Anal. Appl. 4(4), 45-46 (2012))

doi:10.1186/1687-1812-2013-187

Cite this article as: Patel et al.: Some discussion on the existence of common fixed points for a pair of maps. Fixed Point Theory and Applications 2013 2013:187. 